\section{Somatic Embryogenesis and Plant Regeneration from Immature Persimmon (Diospyros kaki Thunb.) Embryos}

\author{
Akira Sugiura ${ }^{1}$ \\ Laboratory of Pomology, Department of Bioproduction Sciences, Ishikawa \\ Prefectural University, Nonoichi, Ishikawa 921-8836, Japan
}
Yoshiko Matsuda-Habu, Mei Gao, Tomoya Esumi, and Ryutaro Tao Laboratory of Pomology, Graduate School of Agriculture, Kyoto University, Sakyo, Kyoto 606-8502, Japan

\begin{abstract}
Additional index words. adventitious bud formation, globular embryo, torpedo-type embryo, embryogenic callus, mass propagation
\end{abstract}

\begin{abstract}
In persimmon, plant regeneration from cultured cells usually takes place through adventitious bud formation. If somatic embryogenesis were possible, the efficiency of mass propagation and genetic engineering would be greatly improved. We attempted to induce somatic embryogenesis from immature embryos and plant regeneration from the induced embryos. Hypocotyls and cotyledons from immature 'Fuyu' and 'Jiro' seeds were cultured in the dark in Murashige and Skoog medium solidified with gellan gum and supplemented with 2,4-dichlorophenoxyacetic acid (2,4-D) and 6-benzyladenine (BA) at various concentrations. Callus formation started at $\approx 2$ weeks of culture, and the callus formation rate was highest at 3 or $10 \mu \mathrm{M}$ combinations of 2,4-D and BA. The initially formed calli gradually became brown or black from which white embryogenic calli (EC) appeared secondarily. After $\approx 8$ weeks of culture, globular embryos were formed from these EC, and the formation proceeded until 20 weeks of culture. Formation of globular embryos was higher with 'Fuyu' than 'Jiro', especially with hypocotyls. When EC with globular embryos were transferred to fresh medium with no plant growth regulators, $\approx 70 \%$ developed to the torpedo-type embryo stage in 6 weeks. The torpedo-type embryos thus formed were germinated and rooted in agar medium with or without zeatin in several weeks without entering dormancy. After germination and rooting, the plantlets were transferred to the same medium and acclimatized for another 4 weeks. As the embryos germinated and rooted simultaneously, the plantlets were easy to grow in pots without transplanting shock. This is the first report on plant regeneration through somatic embryogenesis of persimmon.
\end{abstract}

Persimmon, or kaki (Diospyros kaki), has been widely grown in Japan since ancient times and is consumed as fresh or dried fruit. Because this plant seldom roots by cutting, practical propagation of selected cultivars has been exclusively accomplished by grafting on seedling stocks (Kitagawa and Glucina, 1984). However, seedling stocks are genetically heterozygous, and the performance of trees grafted on such stocks is variable (Tao and Sugiura, 1992b).

Micropropagation would be useful to produce not only uniform self-rooted trees, but also clonal stocks with specific traits (e.g., dwarfing, pest and disease resistance). Much information is available on persimmon organ,

Received for publication 29 June 2007. Accepted for publication 31 Aug. 2007.

This research was supported by a Grant-in-Aid (no. 18580030) for Scientific Research (C) to Akira Sugiura from the Japan Society for the Promotion of Science (JSPS).

${ }^{1}$ To whom reprint requests should be addressed; e-mail sugiura@ishikawa-pu.ac.jp tissue, and cell culture (Cooper and Cohen, 1984; Fukui et al., 1989; Tao and Sugiura, 1992b; Yokoyama and Takeuchi, 1976). Shoot tip culture of current-year shoots is possible (Sugiura et al., 1986) as is plant regeneration from calli and cell cultures derived from dormant buds (Tao et al., 1988; Tao and Sugiura, 1992a). Using these methods, however, the rate of shoot regeneration is low and the resultant shoots are difficult to root depending on their genotype (Murayama et al., 1989). Thus, micropropagation of persimmon is not yet practical.

In persimmon, plant regeneration from cultured tissues or cells always takes place through adventitious bud formation (Tao et al., 1988, 1992, 1997; Tao and Sugiura, 1992a). Only one report is available on somatic embryogenesis from leaf segments of persimmon (Fukui et al., 1988), but these results are questionable because they have not yet been reproduced. If plant regeneration from somatic embryogenesis were possible, the efficiency of mass propagation would be greatly improved. In addition, to develop an effective genetic transformation system, high-frequency plant regeneration through somatic embryogenesis is essential. However, all our attempts to induce somatic embryogenesis from selected materials in the adult phase have so far failed. Thus, in this study, we tried inducing somatic embryogenesis using immature persimmon embryos and regeneration of plantlets from the induced embryos because somatic embryogenesis is usually easier to induce from embryonic organs. If we were successful, we could modify the protocol and finally apply it to adult materials.

\section{Materials and Methods}

Plant materials. Fruits of two nonastringent-type cultivars, 'Fuyu' and 'Jiro', were used. In early September, immature fruits at the later stage of the second fruit growth period were picked from mature trees growing in the Kyoto University orchard. Because the source trees were interplanted with many other cultivars carrying staminate flowers, they produced mostly seeded fruits. After removing calyx lobes, the fruits were washed in running tap water and then immersed in a sodium hypochlorite solution containing $1 \%$ available chloride and $0.1 \%$ Tween 20 for $15 \mathrm{~min}$ followed by three washes with sterile water.

Induction of callus and embryogenesis. Embryos were aseptically excised from immature seed. The cotyledons and hypocotyls were dissected with a sterile surgical knife and then plated separately on $10 \mathrm{~mL}$ of solidified medium in 6-cm-diameter plastic dishes. Murashige and Skoog's basal (MS) medium (Murashige and Skoog, 1962) was used for a series of cultures supplemented with a combination of plant growth regulators (PGRs), i.e., 6-benzyladenine (BA) and 2,4-dichlorophenoxyacetic acid (2,4-D), at different concentrations. The medium containing $3 \%(\mathrm{w} / \mathrm{v})$ sucrose was solidified with $0.3 \%(\mathrm{w} / \mathrm{v})$ gellan gum. The $\mathrm{pH}$ of the medium was adjusted to 5.7 with $1 \mathrm{~N} \mathrm{NaOH}$ before autoclaving at $121{ }^{\circ} \mathrm{C}$ and $1.2 \mathrm{~kg} / \mathrm{cm}^{2}$ for $15 \mathrm{~min}$. The cultures were maintained in the dark at $27{ }^{\circ} \mathrm{C}$ to induce callus formation and embryogenesis. The effects of all possible combinations of BA $(3,10$, and $30 \mu \mathrm{M})$, 2,4-D (3, 10, and $30 \mu \mathrm{M})$, cultivars ('Fuyu' and 'Jiro') and explant types (cotyledon and hypocotyl) on callus and somatic embryo formations were investigated after 20 weeks of culture. Twenty explants were used in each combination.

Germination and rooting. After globular embryos formed on a callus developed to the torpedo stage, they were transferred to 100 $\mathrm{mL}$ Erlenmeyer flasks containing $30 \mathrm{~mL}$ MS medium with or without the PGR zeatin. The medium contained $3 \%(\mathrm{w} / \mathrm{v})$ sucrose and was solidified with $0.8 \%(\mathrm{w} / \mathrm{v})$ agar. $\mathrm{pH}$ adjustment and autoclaving of the culture medium were conducted as described previously. The cultures were also maintained in the dark at $27{ }^{\circ} \mathrm{C}$. Thirty embryos were used in each experiment.

Raising plantlets. After 6 weeks of culture, the torpedo-type embryos were transferred to 
culture tubes $(40 \mathrm{~mm} \varphi \times 130 \mathrm{~mm})$ containing $50 \mathrm{~mL}$ of the same medium. Germinated embryos with radicles were selected and transplanted into fresh medium, and the cultures were maintained in an incubator at $25{ }^{\circ} \mathrm{C}$ under a 16 -h light/8-h dark photoperiod. After $\approx 4$ weeks of culture, the plantlets were transplanted into polycarbonate plastic boxes $(75 \mathrm{~mm} \times 75 \mathrm{~mm} \times 100 \mathrm{~mm})$ containing vermiculite and acclimatized for $\approx 4$ more weeks at $25{ }^{\circ} \mathrm{C}$ under a 14 -h light/10-h dark photoperiod before potting.

Statistical analysis. We calculated 95\% confidence intervals of the globular embryo development, embryo germination, and embryo rooting with binomial distribution (Freund, 1952). Analysis of variance (ANOVA) was performed to analyze significance of the main effects and interactions of the factors on globular embryo formation after the arcsine transformation of percentage data.

\section{Results}

Preliminary experiments. Using immature seeds of 'Fuyu', preliminary experiments were conducted to find effective culture conditions for inducing embryogenesis such as nitrate concentration, type of PGRs, and gelling agents included in the medium. We found that: 1) standard MS salts were more effective than those in which the nitrate concentration was reduced to half strength; 2) the PGRs 2,4-D and BA were more effective than indoleacetic acid (IAA) and zeatin; and 3) gellan gum was better than agar. Auxin IAA never induced embryogenesis. Based on these preliminary results, more detailed experiments were conducted to induce embryogenesis.

Callus formation and embryogenesis. In both hypocotyls and cotyledons of the two cultivars, callus formation began at the cut surface $\approx 2$ weeks after plating in vitro. Combinations of 2,4-D and BA at 3 and 10 $\mu \mathrm{M}$ induced calli at the highest rate $(85 \%$ to $100 \%$ ), whereas either PGR at $30 \mu \mathrm{m}$ tended to suppress callus formation. The lowest formation rate in 'Fuyu' (35\%) occurred when both 2,4-D and BA were at $30 \mu \mathrm{M}$. The growth of the callus initially formed on the original explant was not very vigorous and it gradually became brown or black; a white or yellowish white embryogenic callus formed secondarily on the brown-black callus (Fig. 1). After 8 weeks of culture, globular embryos began to form from these secondary calli. The formation of globular embryos proceeded until $\approx 20$ weeks of culture (Fig. 2). However, no globular embryos developed to the torpedo stage and many of them turned brown and died. Because ANOVA indicated that the main effect of 2,4-D and interactions of 2,4-D with other factors were nonsignificant regardless of the explant types, cotyledon, and hypocotyl (data not shown), we pooled the data obtained with different concentrations of 2,4-D as replications and conducted three-way ANOVA with three factors, BA concentrations, cultivars, and explant types (Table 1). It appeared that 'Fuyu' calli

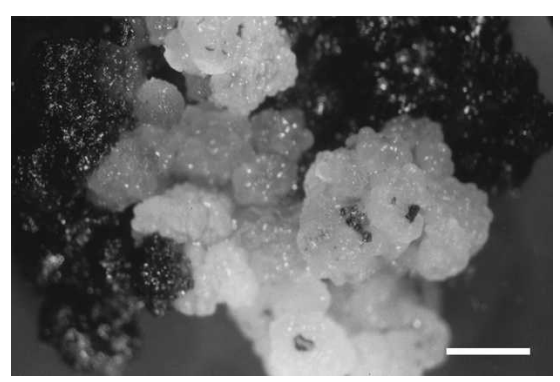

Fig. 1. Embryogenic callus formed secondarily on the brown-black callus of 'Jiro'. Bar $=1 \mathrm{~mm}$.

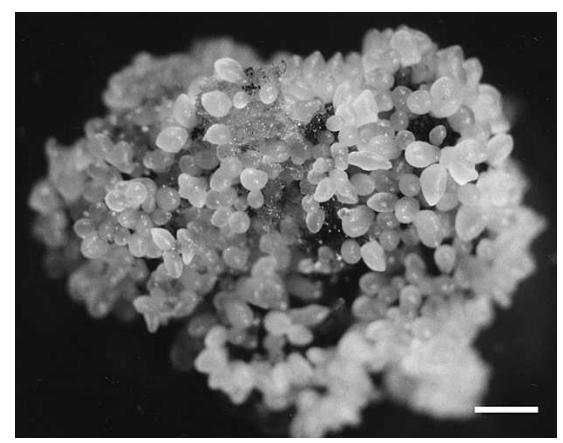

Fig. 2. Globular embryos formed from the embryogenic callus of 'Fuyu'. Bar $=1 \mathrm{~mm}$.

formed more globular embryos than 'Jiro' calli, especially in hypocotyls at $3 \mu \mathrm{M}$ BA (Table 1).

When calli with globular embryos were cut to an appropriate size and transferred to fresh MS medium with no PGR, they developed to the torpedo stage (Fig. 3). In both cultivars, $\approx 70 \%$ of calli with globular embryos formed torpedo embryos (Fig. 4). In contrast, when calli with globular embryos were transferred to fresh MS medium supplemented with 2,4-D and BA, most globular embryos did not develop further and remained at the same stage (Figs. 4 and 5).

Germination and rooting from torpedotype embryos. Torpedo-type embryos were transplanted to agar medium supplemented with 0 or $3 \mu \mathrm{M}$ zeatin. Progressive development from a torpedo embryo to a complete plantlet with shoot and root is shown in Figure 6. After 1 week of culture, $50 \%$ of 'Fuyu' embryos germinated. Germination and rooting rates are shown in Figure 7 . The germination rate was higher in zeatinfree medium than in zeatin medium. Approximately $90 \%$ of embryos germinated after 6 weeks of culture. The rooting rate was also higher in zeatin-free medium than in zeatin medium. A similar trend was observed with 'Jiro' embryos (Fig. 7).

Raising plantlets. Germinated and rooted embryos were removed from the agar medium and transplanted to fresh agar medium with or without zeatin. Plantlets from the embryos grew vigorously on either medium. The shoot stem of plantlets supplemented with zeatin tended to thicken, and their leaves were thicker and darker in color,
Table 1. Effect of 6-benzyladenine, type of explants, and cultivars on globular embryo formation $(\%)$.

\begin{tabular}{lllll}
\hline & \multicolumn{2}{c}{ 'Fuyu' } & \multicolumn{2}{c}{ 'Jiro' } \\
\cline { 2 - 3 } \cline { 3 - 5 } BA $(\mu \mathrm{M})$ & \multicolumn{1}{c}{$\mathrm{C}^{\mathrm{z}}$} & $\mathrm{H}^{\mathrm{z}}$ & $\mathrm{C}^{\mathrm{z}}$ & $\mathrm{H}^{\mathrm{z}}$ \\
\hline 3 & $38 \pm 15^{\mathrm{y}}$ & $58 \pm 16^{\mathrm{y}}$ & $20 \pm 5^{\mathrm{y}}$ & $28 \pm 3^{\mathrm{y}}$ \\
10 & $17 \pm 13$ & $33 \pm 19$ & $8 \pm 3$ & $17 \pm 10$ \\
30 & $10 \pm 0$ & $12 \pm 8$ & $3 \pm 3$ & $3 \pm 3$ \\
\multicolumn{2}{c}{ Analysis of variance } & & &
\end{tabular}

Analysis of variance

\begin{tabular}{lc} 
& $\begin{array}{c}\text { Significance } \\
\text { BA (B) }\end{array}$ \\
Cultivar (C) & $* *$ \\
Explant type (E) & $*$ \\
$\mathrm{~B} \times \mathrm{C}$ & NS \\
$\mathrm{B} \times \mathrm{E}$ & NS \\
$\mathrm{C} \times \mathrm{E}$ & NS \\
$\mathrm{B} \times \mathrm{C} \times \mathrm{E}$ & NS \\
\hline
\end{tabular}

${ }^{\mathrm{z}} \mathrm{C}=$ cotyledon, $\mathrm{H}=$ hypocotyl.

${ }^{\mathrm{y}}$ Mean $\pm \mathrm{SD}$.

Ns,**,*Nonsignificant or significant at $P<0.01,0.05$, respectively.

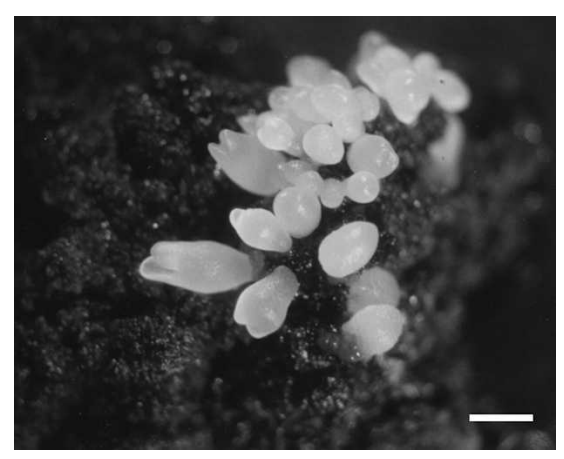

Fig. 3. The torpedo embryos developed from the globular embryos of 'Fuyu'. Bar $=1 \mathrm{~mm}$.

but overall shoot growth seemed better on zeatin-free medium than on zeatin medium. After $\approx 4$ weeks of culture, plantlets were removed from agar medium and transplanted to plant boxes with vermiculite to acclimatize before potting. They showed normal seedling growth (Fig. 8).

\section{Discussion}

Two types of somatic embryogenesis, i.e., direct somatic embryogenesis on explants and indirect somatic embryogenesis through callus formation, are dependent on plant species or the type of explant. The latter type seems more common than the former type, and somatic embryogenesis in persimmon is of the latter type.

The choice of organs or tissues used to induce embryogenic callus formation and embryogenesis is of primary importance. In persimmon, we tried to induce embryogenic calli using various parts of vegetative organs, but all failed except for embryos from immature seeds. Calli derived from vegetative tissues such as primordial leaves in dormant buds (Tao et al., 1988; Tao and Sugiura, 1992a), endosperm (Tao et al., 1997), and anther tissues (Tao et al., 1992) always induce adventitious bud formation. These 


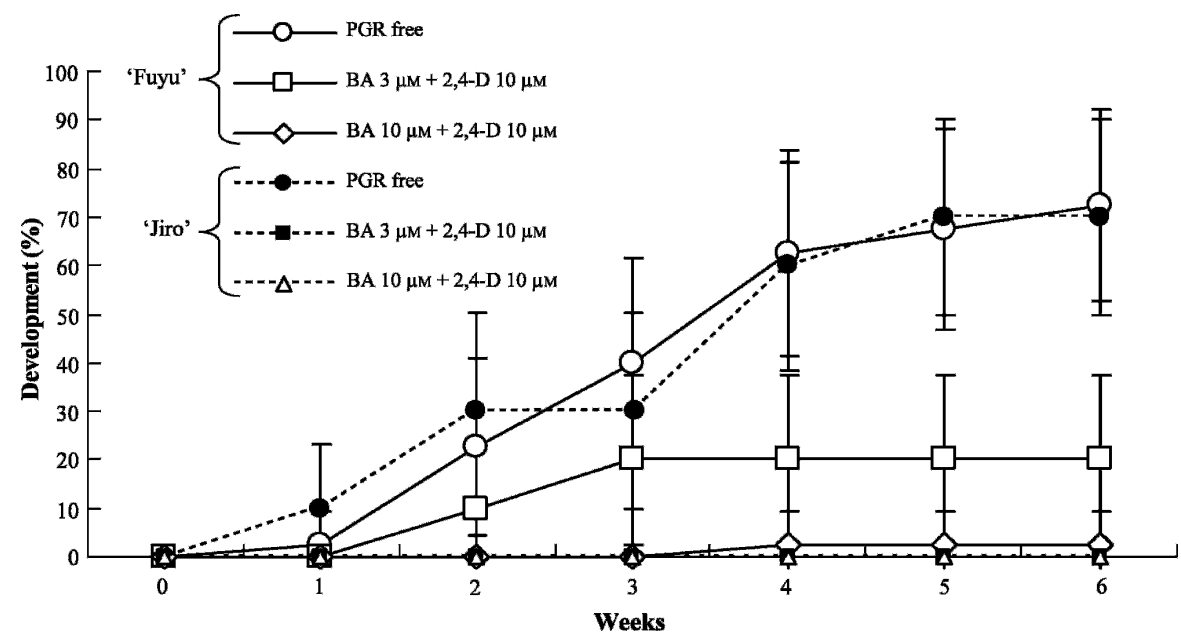

Fig. 4. Percentages of the globular embryos developing to the torpedo stage. Vertical bars represent $95 \%$ confidence intervals that were calculated based on binomial distribution.

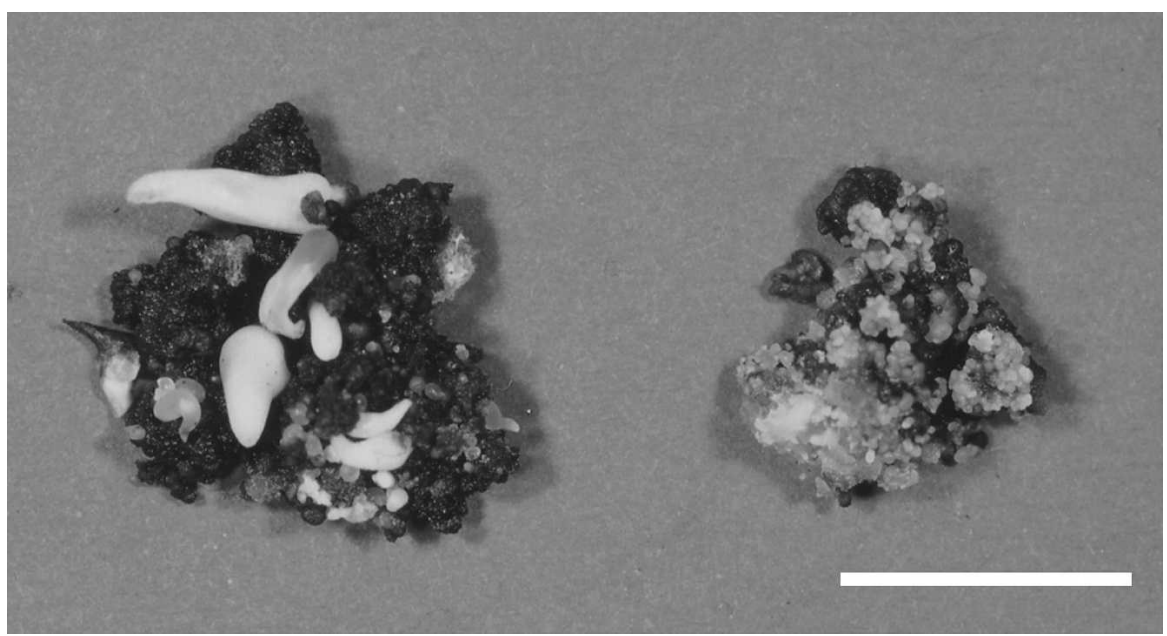

Fig. 5. Embryo development of 'Fuyu' on the medium with no plant growth regulators (left) and the medium supplement with 2,4-dichlorophenoxyacetic acid and 6-benzyladenine (right). Bar $=1 \mathrm{~cm}$.

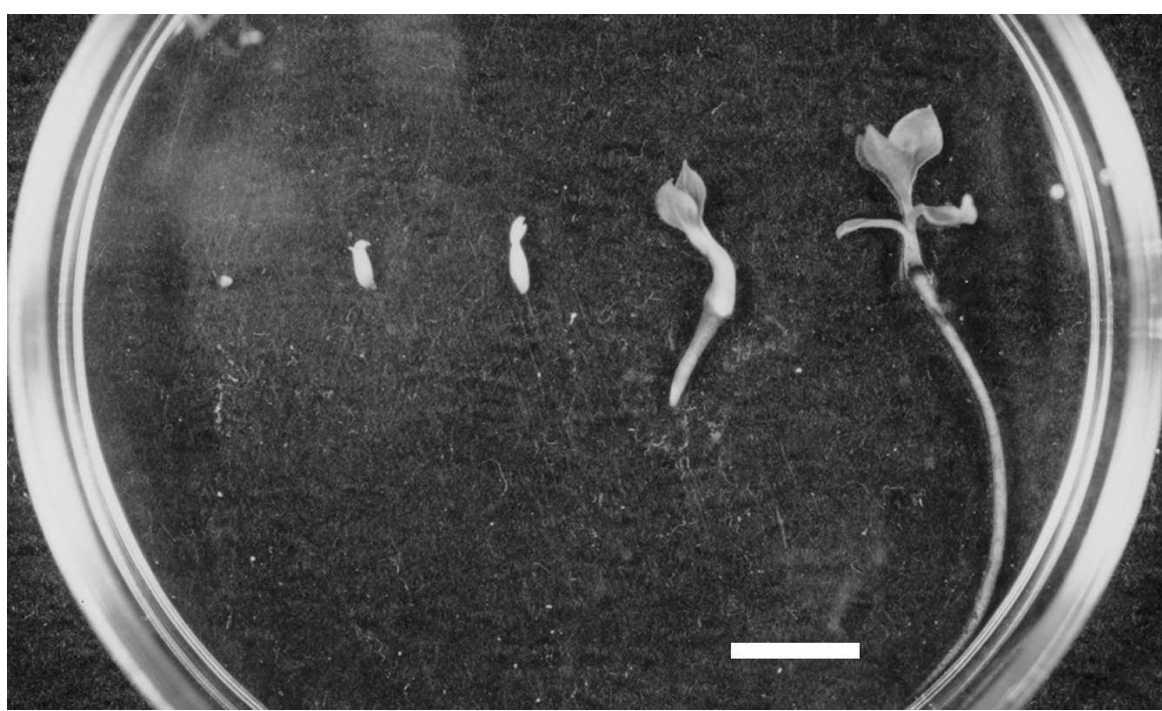

Fig. 6. Embryo development of 'Fuyu' from a torpedo embryo to a complete plantlet with shoot and root. Bar $=1 \mathrm{~cm}$. results imply that embryogenesis may only be intrinsic to embryogenic tissues.

Embryogenic calli from persimmon did not form directly on the explant but appeared secondarily on the initial callus. Initial calli did not grow vigorously and gradually turned brown or black followed by the appearance of a friable embryogenic callus. Similar results were reported for grapes in which the initial callus stopped growing and turned brown before an embryogenic callus appeared (Gray, 1992).

The type of PGRs included in the medium is a very important factor when inducing embryogenic callus formation and embryogenesis. To induce adventitious bud formation from a callus, zeatin and IAA were very effective (Tao et al., 1988; Tao and Sugiura, 1992a), but BA and 2,4-D were more effective than zeatin and IAA at inducing an embryogenic callus. IAA never induced an embryogenic callus.

PGRs were necessary to induce embryogenic callus formation, but once embryogenesis started, the regulators had to be withdrawn from the medium to promote further embryogenesis, resulting in the formation of torpedo-type embryos. When embryogenic calli with globular embryos were subcultured in a medium with BA and 2,4-D, they maintained their embryogenic ability longer for more than 1 year. These calli could resume embryogenesis any time if they were transferred to a medium devoid of PGRs.

In other species, somatic embryos thus obtained are often reported to experience dormancy, and germination usually requires some measures to break the dormancy (Gray, 1992; Gray and Mortensen, 1987; Takeno et al., 1983). In persimmon, however, when torpedo-type embryos were transferred to agar medium without growth regulators, they naturally germinated within several weeks and rooted simultaneously without entering dormancy. In the case of shoots derived from adventitious buds, rooting treatment is required, and rooting is difficult depending of genotype. In addition, even if rooting is successful, transplantation problems are often encountered. Therefore, plantlets derived from somatic embryogenesis are easier to establish compared with plantlets from adventitious shoot formation. Because plantlets through embryogenesis derived from immature embryos are not true to type and genetically variable but easily and efficiently obtainable, they could be a useful source for stock materials in the conventional propagation in the case of limited supply of stock seeds.

In conclusion, we induced somatic embryogenesis and plant regeneration from juvenile embryonic tissue acquired from immature seeds. From the practical viewpoint of mass propagation and genetic engineering of selected persimmon genotypes, however, development of a plant regeneration system through somatic embryogenesis using adult tissues is crucial. Such a breakthrough may be difficult. 


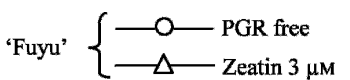

'Jiro' $\left\{\begin{array}{l}\cdots \cdots-\text { PGR free } \\ \cdots \cdots-\cdots \text { Zeatin } 3 \mu \mathrm{M}\end{array}\right.$
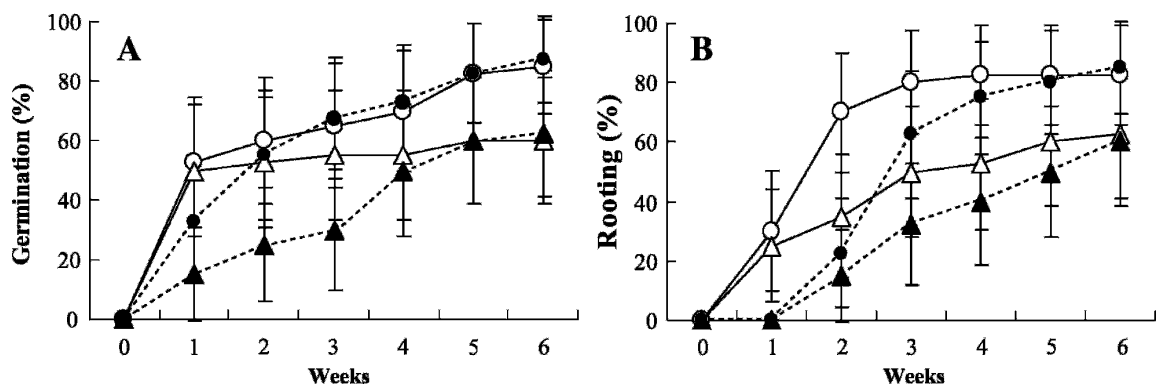

Fig. 7. Percentages of germination (A) and rooting (B) of somatic embryos. Vertical bars represent $95 \%$ confidence intervals that were calculated based on binomial distribution.

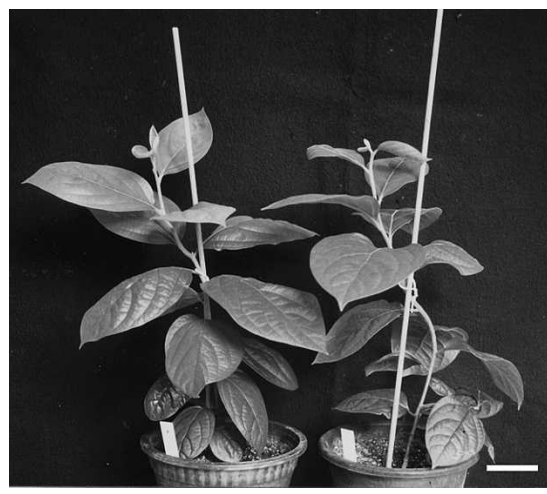

Fig. 8. The plantlets derived from the somatic embryos induced from immature cotyledon of 'Fuyu'. Bar $=10 \mathrm{~cm}$.

\section{Literature Cited}

Cooper, P.A. and D. Cohen. 1984. Micropropagation of Japanese persimmon, Diospyros kaki. Proc. Intl. Plant Prop. 34:118-124.
Freund, J.E. 1952. Modern elementary statistics. Prentice-Hall, Inc., Englewood Cliffs, NJ.

Fukui, H., K. Nishimoto, J. Murase, and M. Nakamura. 1988. Somatic embryogenesis from the leaf tissues of continuously subcultured shoots in Japanese persimmon (Diospyros kaki Thunb.). Jpn. J. Breeding 38:465-469.

Fukui, H., M. Sugiyama, and M. Nakamura. 1989. Shoot tip culture of Japanese persimmon (Diospyros kaki Thunb.). J. Jpn. Soc. Hort. Sci. 58:43-47.

Gray, D.J. 1992. Somatic embryogenesis and plant regeneration from immature zygotic embryos of muscadine grape (Vitis rotundifolia) cultivars. Amer. J. Bot. 79:542-546.

Gray, D.J. and J.A. Mortensen. 1987. Initiation and maintenance of long term somatic embryogenesis from anthers and ovaries of Vitis longii 'Microsperma'. Plant Cell. Tiss. Org. Cult 9:73-80.

Kitagawa, H. and P.G. Glucina. 1984. Persimmon culture in New Zealand. Science Information Publishing Centre, DSIR, Wellington, New Zealand. p. 1-74.

Murashige, T. and F. Skoog. 1962. A revised medium for rapid growth and bioassays with tobacco tissue cultures. Physiol. Plant. 15:473497.

Murayama, H., R. Tao, T. Tanaka, and A. Sugiura 1989. In vitro shoot proliferation and rooting of several Japanese persimmon cultivars [in Japanese with English summary]. J. Jpn. Soc. Hort. Sci. 58:55-61.

Sugiura, A., R. Tao, H. Murayama, and T. Tomana. 1986. In vitro propagation of Japanese persimmon. HortScience 21:1205-1207.

Takeno, K., M. Koshioka, R.P. Pharis, K. Rajasekaran, and M.G. Mullins. 1983. Endogeneous gibberellin-like substances in somatic embryos of grape (Vitis vinifera $\times$ Vitis rupestris) in relation to embryogenesis and chilling requirement for subsequent development of mature embryos. Plant Physiol. 73: 803-808.

Tao, R., W. Ito, and A. Sugiura. 1992. Adventitious bud formation on callus derived from anthers of persimmon 'Meotogaki' and isozyme variations observed in the regenerated plantlets [in Japanese with English summary]. J. Jpn. Soc. Hort. Sci. 61:527-533.

Tao, R., H. Murayama, K. Moriguchi, and A. Sugiura. 1988. Plant regeneration from callus cultures derived from primordial leaves of adult Japanese persimmon. HortScience 23:10551056.

Tao, R., K. Ozawa, M. Tamura, and A. Sugiura. 1997. Dodecaploid plant regeneration from endosperm culture of persimmon (Diospyros kaki L.). Acta Hort. 436:119-128.

Tao, R. and A. Sugiura. 1992a. Adventitious bud formation from callus cultures of Japanese persimmon. HortScience 27:259-261.

Tao, R. and A. Sugiura. 1992b. Micropropagation of Japanese persimmon (Diospyros kaki L.), p. 424-440. In: Y.P.S. Bajaji (ed.). Biotechnology in agriculture and forestry. Vol. 18. High-Tech and Micropropagation II. Springer-Verlag, New York.

Yokoyama, T. and M. Takeuchi. 1976. Organ and plantlet formation from callus in Japanese persimmon (Diospyros kaki). Phytomorphology $9: 273-275$. 